\title{
Preamble for the special issue of amorphous materials
}

\author{
Wei-hua Wang ${ }^{1}$
}

Published online: 29 June 2018

(c) China Iron and Steel Research Institute Group 2018

Amorphous materials are new metal-alloy materials developed in compliance with modern metallurgical rapid solidification technologies and design concepts. They are metal materials which do not have long-range structure orders and cannot be understood and described by means of classical paradigm and theory of solid physics and material sciences. Because of the strong correlation of atoms in the amorphous alloy materials, they have extreme and exotic macroscopic physical properties, such as glass transition, excellent mechanical properties (strength close to theoretical limit), chemical properties (e.g., corrosion resistance), localized rheology and complex dynamic behaviors. They cause challenges to traditional metal physics and theoretical framework of materials. Consequently, amorphous alloy is always a cutting-edge topic for the research of material sciences and technologies. The bulk amorphous alloy materials developed in recent years are significantly superior to conventional metal materials in many aspects, such as strength, hardness, toughness, superplastic forming, soft magnetism, wear resistance, corrosion resistance and radiation resistance. They have important application prospects in the fields of high technology, national defense, information technology and energy. Amorphous alloy materials have also opened a new chapter in the study of amorphous physics.

However, the methods for exploring amorphous materials are different from the traditional methods of exploring materials. The traditional methods of exploring new materials are to obtain new materials mainly by changing and adjusting chemical composition, the structure and phase, and the structure defects. Amorphous materials are new materials obtained by adjusting the "order" or "entropy" of materials. For example, amorphous alloys are high-performance alloys obtained by introducing "structural disorder" through rapid solidification or mixing of different elements. New materials with unique properties can also be obtained by changing and

Wei-hua Wang

whw@iphy.ac.cn

1 Institute of Physics, Chinese Academy of Sciences, Beijing 100190, China adjusting the "chemical order." Disordered high-entropy alloy is a new material obtained in recent years by introducing "chemical disorder" through multi-component mixing. This method of adjusting the material configuration ("order" or "entropy") will lead to the discovery of more similar new amorphous materials, and therefore, its basic physical mechanism deserves more attention and intensive study.

China has been at the forefront of the world in many aspects of amorphous alloy materials. Under the organization of the National Natural Science Foundation of China, an industry-university-research group is established to focus on the core scientific and technical challenges, and a lot of progress has been made in the recent years. In order to help readers understand the latest development in the field of amorphous alloy materials, to promote the further study of amorphous materials, we have arranged the special issue titled "Progress in the Research of Amorphous Materials" and invited some experts who are active in the frontier of this field to introduce the latest progress, problems, status quo and outlook for the amorphous alloy materials from different perspectives in terms of materials, mechanics, magnetism, experiments and theories. We expect to promote the further cooperation and development for the amorphous alloy materials in China.

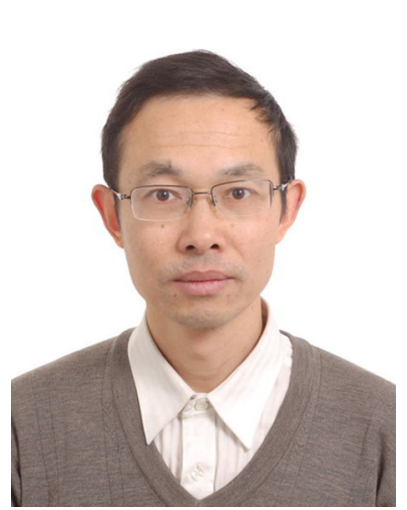

Wei-hua Wang is a professor in Institute of Physics and University of Chinese Academy of Sciences. Prof. Wang, an academician of Chinese Academy of Sciences and TWAS, serves as the head of Extreme Condition Physics Laboratory of Chinese Academy of Sciences and group leader of Amorphous Physics and Materials. He got his Ph.D. degree in condensed matter physics from Institute of Physics, Chinese Academy of Sciences, in 1993. He is Alexander von Humboldt Fellow in Hahn-Meitner Institute in Berlin, Germany. His group focuses his research work on formation, structure and physical properties of metastable materials such as bulk metallic glasses and nanocrystalline materials. 